\title{
Apport scientifique, éducatif et ludique de l'espace Blaise Pascal au muséum Henri-Lecoq de Clermont-Ferrand
}

Claire Bernard, Aurélie Del Prete, Éliane Garmy, Amandine Schmaltz et Nathalie Vidal

\section{Q OpenEdition}

\section{Journals}

Édition électronique

URL : http://journals.openedition.org/ocim/1342

DOI : $10.4000 /$ ocim. 1342

ISSN : 2108-646X

Éditeur

OCIM

Édition imprimée

Date de publication : 25 mars 2014

ISSN : 0994-1908

Référence électronique

Claire Bernard, Aurélie Del Prete, Éliane Garmy, Amandine Schmaltz et Nathalie Vidal, « Apport scientifique, éducatif et ludique de l'espace Blaise Pascal au muséum Henri-Lecoq de ClermontFerrand », La Lettre de I'OCIM [En ligne], 152 | 2014, mis en ligne le 17 avril 2016, consulté le 19 avril 2019. URL : http://journals.openedition.org/ocim/1342 ; DOI : 10.4000/ocim.1342 


\title{
Apport scientifique, éducatif et ludique de l'espace Blaise Pascal au muséum Henri-Lecoq de Clermont-Ferrand
}

\author{
Claire Bernard, Aurélie Del Prete, Éliane Garmy, \\ Amandine Schmaltz et Nathalie Vidal *
}

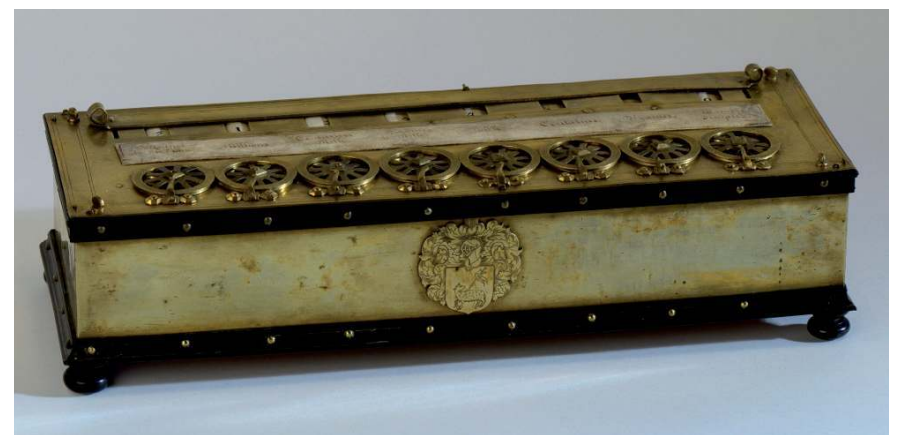

La machine de Marguerite Périer (c) Ville de Clermont-Ferrand/Muséum Henri-Lecoq/Stéphane Vidal

* Claire Bernard, est chargée de mission,

Aurélie Del Prete, est médiatrice,

Amandine Schmaltz, est médiatrice et chargée de mission sur les collections,

Nathalie Vidal, est responsable du département Histoire des Sciences et Techniques et de l'Inventaire pour la mission PATSTEC Auvergne au muséum Henri-Lecoq de Clermont-Ferrand nvidal@ville-clermont-ferrand.fr

Éliane Garmy est professeur des écoles détachée au muséum Henri-Lecoq
Les responsables chargés de la mise en place d'un espace éducatif et ludique consacré aux travaux de Blaise Pascal dressent un premier bilan de cette expérience en montrant l'intérêt de ce projet - mené par une équipe pluridisciplinaire - dont l'objectif était d'associer explications et apports scientifiques, jeu et pédagogie et qui, en définitive, a contribué au renouvellement des outils et méthodes de travail du service éducatif du musée.

La Ville de Clermont-Ferrand possède dans ses collections deux originaux de la première machine à calculer automatique inventée par Blaise Pascal. Depuis janvier 2013, le muséum Henri-Lecoq a ouvert un espace muséographique consacré à l'histoire des sciences. Une place importante est dédiée à Blaise Pascal autour de la présentation de l'exemplaire original de la machine arithmétique. Le deuxième exemplaire est exposé au musée d'Art Roger-Quilliot (MARQ) où est par ailleurs abordé le penseur, dans ses dimensions littéraire, philosophique et théologique.

Grâce à ces machines, le service des publics a développé un discours scientifique autour de la vie de Blaise Pascal en lien avec les mathématiques contemporaines. Ce discours est accompagné d'une démonstration à l'aide d'une reproduction fonctionnelle. En 2008/2009, le succès de l'exposition temporaire Si la mesure m'était comptée... nous a montré l'intérêt du public clermontois et auvergnat pour les sciences et leur histoire. Les professeurs de mathématiques et de sciences physiques ont porté un intérêt conséquent pour cette thématique en investissant pleinement l'exposition (122 classes, 3200 élèves). Le nouvel espace, présentant l'histoire des sciences, aux échelles locale et nationale, à travers 
de grands scientifiques, offre l'opportunité de développer des activités pédagogiques autour des mathématiques et de les rendre accessibles au plus grand nombre.

\section{Description de l'espace muséographique}

L'espace consacré à Blaise Pascal au muséum HenriLecoq se découpe en 7 parties. Le visiteur est tout d'abord accueilli par la silhouette grandeur nature de Blaise Pascal inspirée d'un marbre réalisé par Claude Ramey en 1824 et exposé au MARQ. La particularité de ce marbre est de présenter Pascal lisant un ouvrage et appuyé de son coude gauche sur une stèle sur laquelle sont représentés symboliquement le profil du Puy-de-Dôme, un baromètre et une cycloïde (courbe décrite par un point d'un cercle roulant sans glisser sur une droite).

\section{Biographie de Blaise Pascal}

La biographie est illustrée du portrait de Blaise Pascal et des portraits des membres de sa famille qui ont beaucoup compté dans sa vie. Elle est volontairement simplifiée et axée sur les événements scientifiques qui ont marqué toute la vie de Pascal. Une frise chronologique souligne certains événements majeurs qui jalonnent l'histoire de France durant la vie de Blaise Pascal afin de permettre de se repérer dans le temps.

\section{Le château de Bien-Assis}

Ce château a été la propriété du beau-frère de Blaise Pascal, Florin Périer, célèbre également pour avoir réalisé l'expérience du Puy-de-Dôme. Aujourd'hui, il ne reste plus que la tour et le porche exposés au Jardin Lecoq situé à quelques mètres du muséum Henri-Lecoq. Son dernier propriétaire fut la manufacture française des pneumatiques Michelin qui réalisa une importante campagne photographique du bâtiment avant sa disparition.

Soucieux de préserver son patrimoine et de mieux le connaître, Michelin a rejoint la mission nationale PATSTEC (Mission nationale de sauvegarde du Patrimoine scientifique et technique contemporain) en 2009. Une convention lie la Ville de Clermont-Ferrand et Michelin afin que le muséum Henri-Lecoq réalise, en étroite collaboration avec le service Patrimoine de Michelin, l'inventaire des instruments. Il consiste à identifier les objets à l'aide d'un numéro d'inventaire, réaliser une campagne photographique et rédiger une fiche d'inventaire. Ces fiches d'inventaire alimentent les bases régionale et nationale du réseau ainsi que celles de la firme.

C'est grâce à ce partenariat que le muséum a pu utiliser les plaques de verre du château et commander un travail de reconstitution virtuelle de celui-ci en 3D. L'idée est de faire connaître cette demeure entière aux



Marbre de Blaise Pascal, 1824, Claude Ramey (1754-1838) (c) Ville de Clermont-Ferrand/MARQ/Rémi Boissau

visiteurs et aux habitants de la région clermontoise en complément des deux vestiges présentés au Jardin Lecoq. Cette demeure est également très importante dans la vie de Blaise Pascal. Il y séjourna à de nombreuses reprises et au lendemain de son décès, c'est là que furent découvertes ses premières pensées cousues dans son vêtement. Plusieurs amis et proches de Blaise Pascal entreprirent de les réunir, c'est ainsi que furent publiées les Pensées de Blaise Pascal huit ans après sa mort.

\section{Blaise Pascal ingénieur}

Le cœur de cet espace est une vitrine présentant l'exemplaire original de la machine arithmétique de Blaise Pascal, mise au point en 1645 après trois ans de recherche. Lobjet, trésor national, est présenté sous une cloche de verre sécurisée et fermée à clé. La machine présentée est la machine de Marguerite Périer (MHLCLFE-138) appelée également «machine de Clermont-Ferrand ». Marguerite Périer était la nièce et filleule de Blaise Pascal. Elle légua par testament à l'Oratoire de Clermont-Ferrand la machine qui se retrouva dans 
les collections de la Ville de Clermont-Ferrand, au lendemain de la Révolution française.

Blaise Pascal inventa cette machine pour répondre à un problème technique rencontré par son père. Ce dernier est collecteur d'impôts et sa charge devient de plus en plus lourde. Il réalise de nombreux et importants calculs mais est confronté à des erreurs. Il répète et recompte inlassablement, s'épuisant à la tâche et délaissant sa famille. Blaise Pascal s'en inquiète et cherche une solution pour aider son père dans ce travail. Il constate que l'erreur de son père porte sur la retenue, oubliée ou décalée. Il va donc concevoir et réaliser la première machine à calculer mécanique fonctionnelle avec report automatique de la retenue.

La machine exposée au muséum servait pour les comptes abstraits, elle possède huit roues à l'inscripteur. Chaque roue est équipée d'une roue étoilée à 10 branches. Il suffit de tourner ces roues étoilées à l'aide d'un stylet, dans le sens des aiguilles d'une montre, pour voir afficher les chiffres au totaliseur. Ce dernier est composé de cylindres qui portent deux séries de chiffres, une série est utilisée pour les additions, l'autre pour les soustractions. Le choix du mode opératoire se fait à l'aide d'une baguette mobile installée sur le totaliseur. Cet original est fonctionnel mais n'est pas utilisé. Pour cela, le muséum possède deux reproductions fonctionnelles (échelle 1). Ces machines permettent de faire des démonstrations pour le public et lors de manifestations comme les Journées du Patrimoine, la Nuit des Musées ou dernièrement la soirée « Voyage sur la math-monde » organisée par le muséum dans le cadre de la semaine nationale des mathématiques.

La présentation de la machine de Marguerite Périer est complétée par une maquette animée du sautoir à l'échelle 10. Toute l'ingéniosité de la machine de Pascal repose sur une pièce maîtresse appelée le sautoir. Lorsque le cylindre est actionné à l'aide de l'inscripteur, le sautoir monte au fur et à mesure jusqu'à atteindre

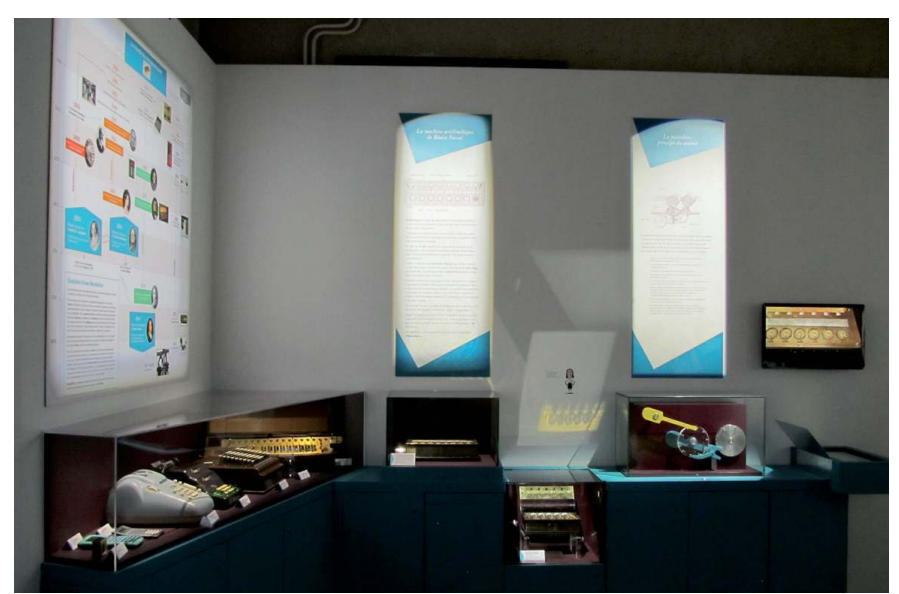

Dans l'Espace Pascal ingénieur, présentation de la machine de Marguerite Périer (c) Ville de Clermont-Ferrand/Muséum Henri-Lecoq/Amandine Schmaltz sa position la plus haute à l'aide de deux tiges décentrées situées entre les engrenages de la machine. La deuxième tige continue à tourner et libère alors le sautoir qui chute. Larrière du sautoir possède une sorte de crochet qui vient attraper un pignon de l'engrenage de la roue suivante. Au moment de la chute, le sautoir pousse ainsi d'un cran le cylindre suivant entraînant le report automatique de la retenue visible alors sur le cylindre.

Lespace consacré à la machine arithmétique est complété par la présentation virtuelle de la machine de Marguerite Périer. Elle permet de voir l'intérieur de la machine tout en la faisant fonctionner. Elle est présentée sur un écran tactile manipulé par les visiteurs. Cette manipulation est projetée en temps réel sur un écran de télévision. Laccès à cette machine virtuelle est un moment privilégié pour le visiteur qui se met en situation de manipuler une machine de Blaise Pascal. Cette première section présente également d'autres machines à calculer, celles issues des collections de la Ville de Clermont-Ferrand, allant du XVII ${ }^{e}$ siècle, avec la machine de Blaise Pascal, jusqu’à la fin du XX' siècle, afin d'illustrer la généalogie de cette invention majeure et son héritage dans les technologies d'aujourd'hui.

\section{Blaise Pascal mathématicien}

Cette partie purement mathématique a été réalisée en étroite collaboration avec des chercheurs et professeurs de l'université Blaise-Pascal et de l'Institut de Recherche pour l'Enseignement des Mathématiques (IREM)

Cet espace présente une partie seulement des travaux mathématiques de Blaise Pascal. On y aborde les probabilités, les cycloïdes et les coniques (courbes planes algébriques). Il est prévu de renouveler cet espace afin de pouvoir y présenter d'autres travaux mathématiques du savant et d'avoir au final des supports pédagogiques et ludiques présentant l'intégralité des travaux mathématiques de Blaise Pascal.

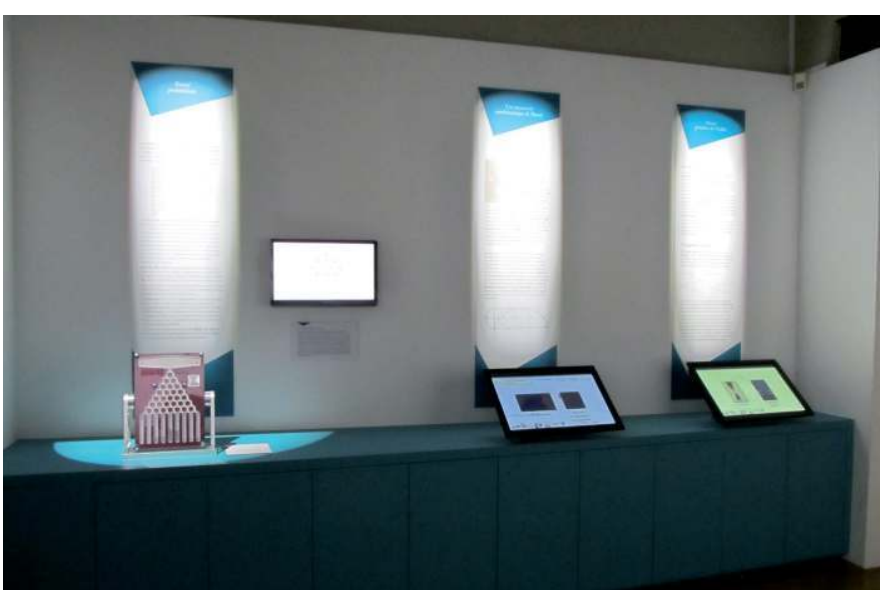

Dans l'Espace Pascal mathématicien, probabilités, triangles arithmétiques, cycloïdes, hexagramme mystique sont abordés de façon ludique et pédagogique. (-) Ville de Clermont-Ferrand/Muséum Henri-lecoq/Amandine Schmaltz 
La difficulté était de rendre compréhensible ces concepts théoriques par le plus grand nombre. La première partie est consacrée aux probabilités. Elles sont introduites par la manipulation d'une planche de Galton qui permet de mettre en évidence la répartition des billes en forme de cloche, et une animation expliquant la réalisation du triangle arithmétique. Les mathématiciens fixent la naissance du calcul des probabilités à l'échange de lettres entre Blaise Pascal et Pierre de Fermat en 1654, au sujet du partage équitable des mises entre 2 puis 3 joueurs s'ils décident d'interrompre une partie en cours. Pascal reprendra la question dans son Traité $d u$ triangle arithmétique. La théorie des probabilités est devenue depuis 50 ans une branche majeure des mathématiques qui entretient des relations fructueuses avec l'analyse, l'algèbre et la géométrie.

Les cycloïdes sont présentées sur un écran tactile : en faisant varier les paramètres, les visiteurs tracent des hypocycloïdes ou des épicycloïdes. Une animation sur le calcul de l'aire d'une cycloïde vient en complément. Pascal a mis au point une méthode originale, combinaison d'arithmétique et de géométrie, pour calculer le volume, la surface ou le centre de gravité des solides de révolution engendrés par une courbe. Pour la mettre à l'épreuve, il décida de l'appliquer à la cycloïde.

Deux animations, toujours sur écran tactile, font découvrir les coniques: l'une présente différentes coniques, l'autre, le jardinier géomètre, aborde le principe de l'hexagramme de Pascal. Dès 1640, Pascal publie son Essay pour les Coniques. Dans ce texte d'une page, il inscrit un hexagone dans un cercle et souligne l'alignement des trois points d'intersection des trois couples de côtés opposés de cet hexagone, puis énonce la généralisation à n'importe quelle conique, Pascal appelait cette configuration « hexagramme mystique ».

\section{Blaise Pascal physicien}

La réalisation de cet espace a également fait appel à un conseil scientifique regroupant professeurs et maîtres de conférences de l'université Blaise-Pascal et de l'Observatoire de Physique du Globe de Clermont (OPGC). On y aborde l'expérience du Puy-de-Dôme sous la forme d'un dessin animé. Blaise Pascal conçoit intellectuellement cette expérience et demande à son beaufrère de la réaliser. Il voulait ainsi démontrer que le phénomène connu à l'époque sous le nom de « suspension des liqueurs » était sans lien avec le postulat d'« horreur du vide » mais bien due à l'existence d'une pesanteur de l'air, la pression atmosphérique, déjà mise en évidence par Évangéliste Torricelli, quelques années plus tôt en 1644.

Lexpérience du Puy-de-Dôme commence au matin du 19 septembre 1648 au pied de l'église de Saint-Pierredes-Minimes sur la place de Jaude (nom actuel) de Clermont-Ferrand, à $350 \mathrm{~m}$ d'altitude. Un premier relevé est fait à l'aide d'un tube de verre rempli de mercure et retourné dans un récipient également rempli de mercure. En présence de plusieurs témoins, le niveau du mercure dans le tube se stabilise à 26 pouces 3 lignes et demi $(71,2 \mathrm{~cm})$. L'expédition se lance à l'assaut du sommet du Puy-de-Dôme et l'expérience est renouvelée à $1450 \mathrm{~m}$ d'altitude. Le niveau du mercure dans le tube se stabilise à 23 pouces et 2 lignes $(62,7 \mathrm{~cm})$ soit près de $8 \mathrm{~cm}$ en-dessous de la valeur obtenue initialement. Florin Périer renouvelle 5 fois l'expérience au sommet du Puy-de-Dôme et confirme le résultat obtenu. La colonne d'air est moins pesante au sommet du Puy-de-Dôme et exerce une pression moindre sur le mercure du récipient. La colonne de ce dernier est alors moins haute dans le tube de verre, ceci afin d'équilibrer les deux forces : pression de la colonne d'air et poids de la colonne de mercure. Blaise Pascal démontre ainsi qu'il se passe quelque chose entre les deux points séparés d'un dénivelé de $1000 \mathrm{~m}$ et met l'accent sur les effets de la pression atmosphérique.

Lespace est complété par une vitrine présentant une série d'instruments utilisés pour mesurer tout type de pression : baromètres pour la mesure de la pression atmosphérique, oscillomètre pour la pression artérielle et manomètre pour la mesure de la pression de différents gaz. Les instruments présentés proviennent de collections diverses : muséum Henri-Lecoq (63), musée Crozatier (43), IUFM d'Auvergne (63), Michelin (63). La sélection des instruments a été réalisée grâce à l'inventaire de la mission PATSTEC.



Dans I'Espace Pascal physicien, I'expérience du Puy-de-Dôme, la vitrine PATSTEC (๑) Ville de Clermont-Ferrand/Muséum Henri-Lecog/Amandine Schmaltz 


\section{La mission PATSTEC en Auvergne}

En 2003, le ministère de la Recherche a chargé le directeur du musée des Arts et Métiers d'une mission nationale de sauvegarde et de valorisation du patrimoine scientifique et technique contemporain. La mission a pour but d'inventorier les instruments scientifiques et techniques marqueurs de la recherche et des développements scientifiques et techniques d'hier, d'aujourd'hui et de demain. La mission a également pour but de conserver la mémoire des chercheurs, témoins, au même titre que les instruments, de l'évolution technique et scientifique. Les objectifs sont de sensibiliser les structures et personnes au patrimoine scientifique et technique, créer un réseau local autour d'un chef de projet, conseiller, proposer des outils communs, réaliser une base de données et un site Internet communs, et valoriser des actions sous diverses formes au niveau national.

La mission PATSTEC Auvergne est coordonnée par la Ville de Clermont-Ferrand/Muséum Henri-Lecoq depuis 2005. Elle a pour but l'inventaire scientifique et technique dans les 4 départements de la Région, de développer une base de données régionale (museelecoq.clermontferrand.fr) qui compte plus de 8000 fiches. À ce jour, la mission régionale a établi de nombreux inventaires et partenariats : université d'Auvergne, université Blaise-Pascal, INRA, BRGM, IFMA, Manufacture française des pneumatiques Michelin, musée Crozatier, de nombreux lycées... et collabore au projet européen ThinkMotion coordonné par l'IFMA pour la France.

\section{Blaise Pascal entrepreneur}

Blaise Pascal participe à la grande campagne de dessèchement du marais poitevin lancée par le roi afin d'agrandir la surface des terres agricoles. Pascal prend part, avec son ami le duc de Roannez, dans la société de François Brisson, sénéchal de Fontenay-le-Comte en mars 1654. En s'engageant dans cette société, Pascal souhaite d'une part gérer sa fortune personnelle et, d'autre part, mettre en pratique ses connaissances et découvertes dans le domaine de l'hydraulique. Il s'intéresse à la statique des eaux mais également à leur mouvement. Le Traité de l'équilibre des liqueurs et de la pesanteur de la masse de l'air, publié en 1663, après la mort de Blaise Pascal, y décrit pompes et siphons, indispensables à l'assèchement de ces terrains inondés. La zone choisie par Brisson présente une très faible pente nécessitant l'utilisation de machines permettant d'élever l'eau. La presse hydraulique inventée par Pascal pouvait y être très utile.
En 1662, Blaise Pascal fonde, avec le duc de Roannez, une société de transport. Le principe est de transporter, suivant un itinéraire défini, des arrêts et horaires fixes, des voyageurs dans des carrosses. Le tarif de la course s'élève à 5 sols. Pascal invente ainsi les premiers transports en commun, les carrosses à 5 sols. Deux panneaux exposent les deux entreprises de Pascal, celui consacré aux carrosses à 5 sols est illustré d'une carte de Paris montrant les 3 premières lignes mises en place.

Le contexte scientifique de l'époque et Port-Royal Une vaste carte de l'Europe présente les principaux personnages qui ont de près ou de loin correspondu, échangé et/ou travaillé avec Blaise Pascal. On y retrouve le père Marin Mersenne (1588-1648), minime, savant et philosophe, qui, par sa gigantesque correspondance, a servi la diffusion des connaissances scientifiques à travers toute l'Europe savante de son temps ; Gilles Personne de Roberval (1602-1675), mathématicien et physicien, qui réalise des démonstrations de la machine arithmétique de Blaise Pascal et encore Christian Huygens (1629-1695) qui entre en contact avec Pascal en 1659, ce dernier lui adresse une des Lettres de A. Dettonville (un des noms de plume de Blaise Pascal) sur la comparaison de l'ellipse et de la cycloïde. L'espace se termine par une évocation de l'abbaye de Port-Royal où Pascal prit le temps de faire une retraite après sa conversion.

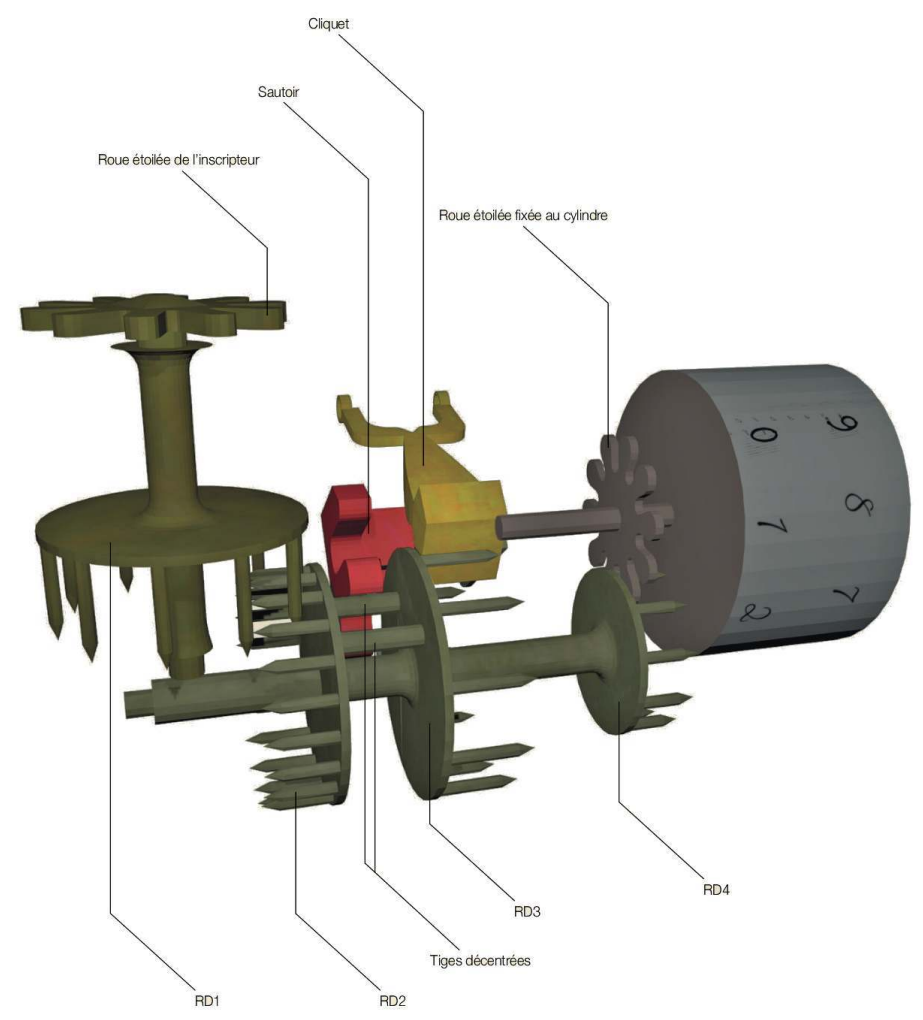

Mécanisme élémentaire de la machine arithmétique de Blaise Pascal (extrait de la machine virtuelle réalisée par Christophe Bascoul/IFMA) 


\section{Démarche scientifique, culture scientifique et Publics pour Pascal mathématicien}

\section{Les scolaires}

L'Éducation nationale souligne l'importance et l'apport de la culture scientifique : les mathématiques, la technologie et les sciences expérimentales font partie de la culture au sens où elles permettent de se construire une représentation globale et cohérente du monde et de mieux comprendre son environnement quotidien. La culture scientifique et technologique repose :

- sur une connaissance des principes et des finalités du raisonnement scientifique,

- sur une pratique effective de la démarche scientifique.

Elle constitue l'une des sept compétences définies par le socle commun que tout élève doit maîtriser en fin de scolarité obligatoire. Réaffirmant l'importance de cette culture, le ministre de l'Éducation nationale a lancé, le 31 janvier 2011, un plan pour les sciences et technologies à l'École.

À la sortie de l'école obligatoire (Pilier 3 du socle commun - Capacités), l'élève doit être en mesure d'appliquer les principes et processus mathématiques de base dans la vie quotidienne. Pour cela, il doit être capable :

- de raisonner logiquement, de pratiquer la déduction, de démontrer ;

- de communiquer, à l'écrit comme à l'oral, en utilisant un langage mathématique adapté ;

- d'effectuer, à la main, un calcul isolé sur des nombres en écriture décimale de taille raisonnable et, à la calculatrice, un calcul isolé sur des nombres relatifs en écriture décimale.

Même s'il y a une approche du nombre dès la fin de la maternelle, les notions de calcul et de nombre apparaissent réellement au cycle 2. Jusqu'au collège, les programmes sont découpés de façon identique : nombres et calcul, géométrie, grandeurs et mesures, organisation et gestion des données.

La calculatrice est un outil qui va devenir récurrent à partir du cycle 3 : utilisation raisonnée en fonction de la complexité des calculs. Jusqu'en classe de Troisième, le calcul et le nombre restent au centre de l'enseignement. C'est en classe de Seconde que le programme va s'articuler autour de la démarche scientifique, l'ordinateur ou la calculatrice devenant un outil de résolution de problèmes.
Le service éducatif du muséum Henri-Lecoq regroupe deux professeurs (primaire et lycée) et les médiatrices du musée. En collaboration avec un conseil scientifique, ce service a élaboré des dossiers pédagogiques, des visites commentées, des parcours-jeux et une mallette s'inscrivant dans le programme de culture scientifique de l'Éducation nationale. Ces activités touchent les classes de la maternelle au lycée. L'objectif du service éducatif est de présenter aux élèves l'aspect ludique des mathématiques pour contrer d'éventuelles appréhensions.

Histoire du calcul en quelques instruments

Le développement de la culture scientifique dans les programmes scolaires et la collection de machines arithmétiques du muséum Henri-Lecoq nous ont conduits à proposer aux classes un atelier de découverte de différents instruments de calcul. Ce dernier, intitulé "Histoire du calcul en quelques instruments ", permet de présenter divers instruments inventés par l'Homme au cours du temps et dans différentes civilisations. Cette approche replace, dans un premier temps, l'invention de Blaise Pascal dans l'évolution des concepts mathématiques à travers les instruments (calculi, bouliers, bâtons de Néper, règles à calcul...). Ainsi nous reprenons ce que sont un chiffre et un nombre, la numération et l'invention du 0, l'origine du calcul avec le dénombrement, les bases 10 (ex. mètre), 20 (ex. système inca) et 60 (ex. heure). L'observation et la manipulation des instruments de calcul d'origines diverses permettent à l'enfant de s'approprier ces notions par l'expérimentation et la déduction.

C'est au médiateur scientifique que revient d'adapter son discours et les instruments présentés en fonction du niveau du groupe. Cette animation peut donc être proposée aussi bien à des classes de cycle 2 qu'à des Terminales scientifiques. Elle a également été adaptée pour une classe pour l'inclusion scolaire (CLIS) de 8 élèves. Cette activité pourrait être aussi développée pour des personnes en difficulté face au calcul et aux nombres (problématique actuelle de l'innumérisme).

Dans un second temps, il s'agit de présenter cette invention dans une perspective historique, politique et sociale. Il s'agit donc de :

- présenter Blaise Pascal, le scientifique ;

- replacer Blaise Pascal et son invention dans son contexte historique ;

- comprendre l'élaboration de la machine arithmétique (fonctionnement, technique et ergonomie) ;

- présenter rapidement les machines arithmétiques et les abaques après Blaise Pascal. En effet, la machine de Pascal est la première d'une longue série de machines à calculer ayant abouti au microprocesseur. 
Des engrenages pour additionner

Il nous paraît important d'aborder l'élaboration technique de la machine. Celle-ci fonctionne grâce à des engrenages qui permettent le passage d'un mouvement horizontal à un mouvement vertical. Mais cette machine répond aussi techniquement à la nécessité de faire passer la retenue automatiquement au rang supérieur.

Les deux originaux et la reproduction fonctionnelle ne mettent pas en valeur les engrenages qui ne sont pas visibles. L'idée d'une reproduction transparente a émergé mais a vite été abandonnée puisqu'il existe une machine virtuelle permettant de voir le fonctionnement des engrenages.

Une mallette pédagogique «Des engrenages pour additionner " a donc été réalisée pour le cycle 3 afin d'approfondir la découverte de ces mécanismes et leur utilisation variée, ainsi que la transmission du mouvement. L'étude des engrenages entre dans les programmes «sciences expérimentales et technologiques-les objets techniques » du cycle 3 : objets mécaniques et transmission du mouvement. La mallette sera mise à la disposition des écoles de la région Auvergne soit en complément d'une visite au musée, soit pour découvrir en classe cette machine à calculer pour les classes qui ne peuvent pas se déplacer.

La mallette a pour but l'apprentissage de la démarche scientifique à partir d'une invention également objet historique : observation, tentative de reproduction, comparaison et déduction devront amener l'élève à la compréhension du montage d'engrenages de la machine de Blaise Pascal. Une ouverture sera ensuite possible vers d'autres objets utilisant des engrenages comme les montres.

Les premières séances reposent sur l'observation et la manipulation des engrenages. L'objectif est de se familiariser avec la transmission et la transformation d'un mouvement. Ces séances d'expérimentation se terminent par une reconstitution partielle des mécanismes à l'œuvre dans la machine de Pascal en mettant en évidence le rôle joué par le sautoir. Une vidéo de la maquette du sautoir en fonctionnement est alors diffusée. Dans un second temps, les élèves manipulent la machine virtuelle sur ordinateur. En un clic, ils enlèvent le caisson et accèdent aux différentes pièces : les roues, les engrenages, les cliquets, les cylindres et les sautoirs, tout en calculant. Il s'agit d'une visite à l'intérieur du mécanisme général, il est possible de voir les engrenages et les autres pièces en action. Lors de cette deuxième séance, le rôle du sautoir est abordé par le jeu et la manipulation virtuelle. Les élèves actionnent la machine virtuelle à leur propre rythme. Ils sont également amenés à tester librement la façon de faire les calculs. Additions et soustractions sont donc abordées de manière ludique. Pour observer le sautoir en action, ils vont

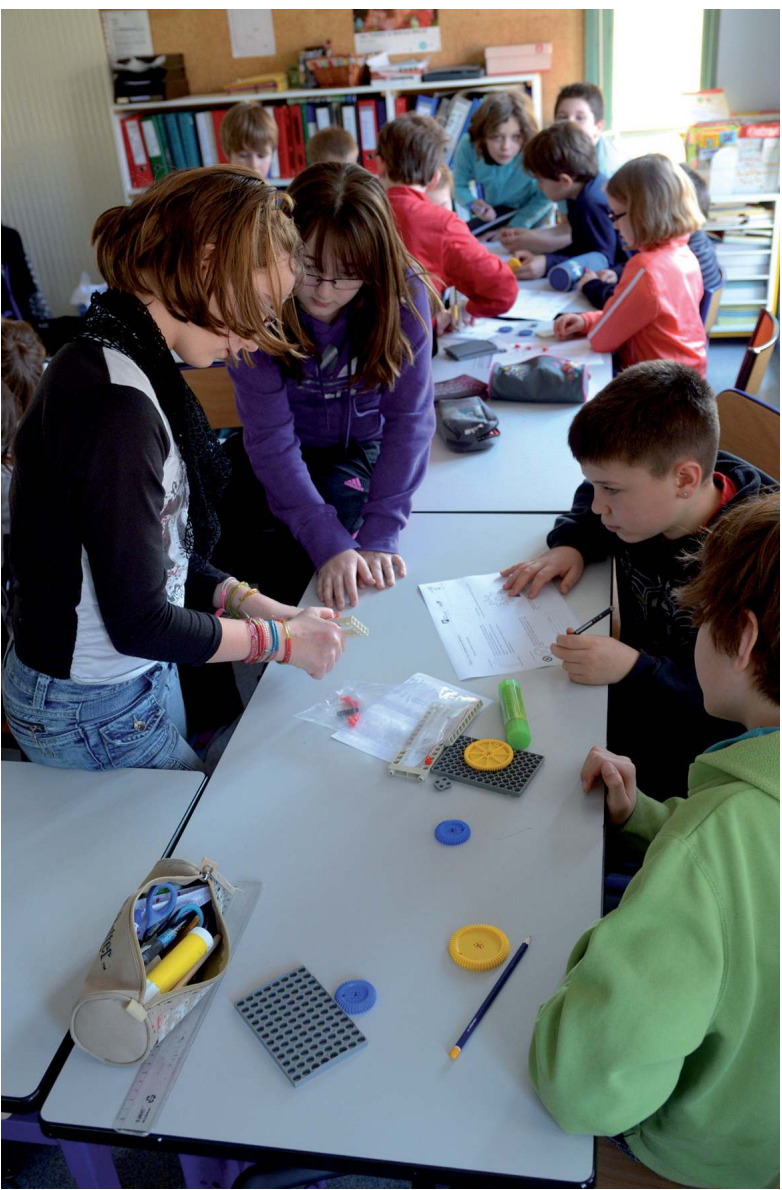

Test de la mallette pédagogique "Des engrenages pour additionner " ๑ Ville de Clermont-Ferrand/Muséum Henri-Lecoq/Stéphane Vidal

devoir faire des opérations impliquant une retenue et pourront réfléchir à son rôle fondamental dans le calcul. En effet, les erreurs de calcul sont liées aux retenues. Le sautoir est donc la pièce maîtresse de la machine.

Les différentes séances présentées dans la mallette ont été testées dans une classe de CM1/CM2. Les élèves se sont montrés enthousiastes. Grâce à leurs questions et leurs remarques, nous avons amélioré la mallette. Pour le professeur, les séances bien cadrées et le matériel mis à disposition ont représenté un atout non négligeable. Cette mallette a été également intégrée au dispositif de formation de la Maison pour la Science en Auvergne, mis en place avec l'IREM : « Pascal, l'homme de science, perspectives historique et patrimoniale », une des quatre formations du projet « Blaise Pascal et la science d'aujourd'hui ». Là encore, les remarques et réflexions des professeurs nous ont permis d'améliorer les fiches d'accompagnement. Des dossiers pour les enseignants et des parcours-découvertes complètent l'offre pédagogique (découverte de 6 autres scientifiques et de leur travail). Nous avons également le projet de développer, pour les écoles primaires, 
des activités autour des cycloïdes, courbes étudiées par Blaise Pascal. Il s’agira de tracer des figures géométriques faisant appel à ces courbes, à l'image du jeu du « spirographe ».

\section{Conclusion : I'ouverture vers d'autres publics}

Si les scolaires sont un public quantitativement important du musée, bien d'autres publics fréquentent nos salles : accueils de loisirs, enfants des ateliers des vacances et des mercredis, adultes seuls ou en groupes. Si l'on entend bien souvent le public dire « je n'aime pas les maths », " je suis nul(le) en maths » ou encore «les maths ne miaiment pas »..., il suffit souvent de dissimuler des exercices mathématiques dans d'autres disciplines pour que ces dernières deviennent compréhensibles, accessibles et parfois faciles. Ainsi, le passage du plan sur quadrillage aux relevés de coordonnées (méthode utilisée pour le Primaire) se fait assez facilement lors des stages de fouilles archéologiques à condition de ne jamais prononcer les mots : mathématiques, repères orthonormés, abscisses, ordonnées... Certains jeunes (collège ou lycée) ne font pas d'euxmêmes la liaison entre leurs cours et l'application pratique sur la fouille. À la question « avez-vous fait des maths pendant le stage ? », la majorité répond non instinctivement.

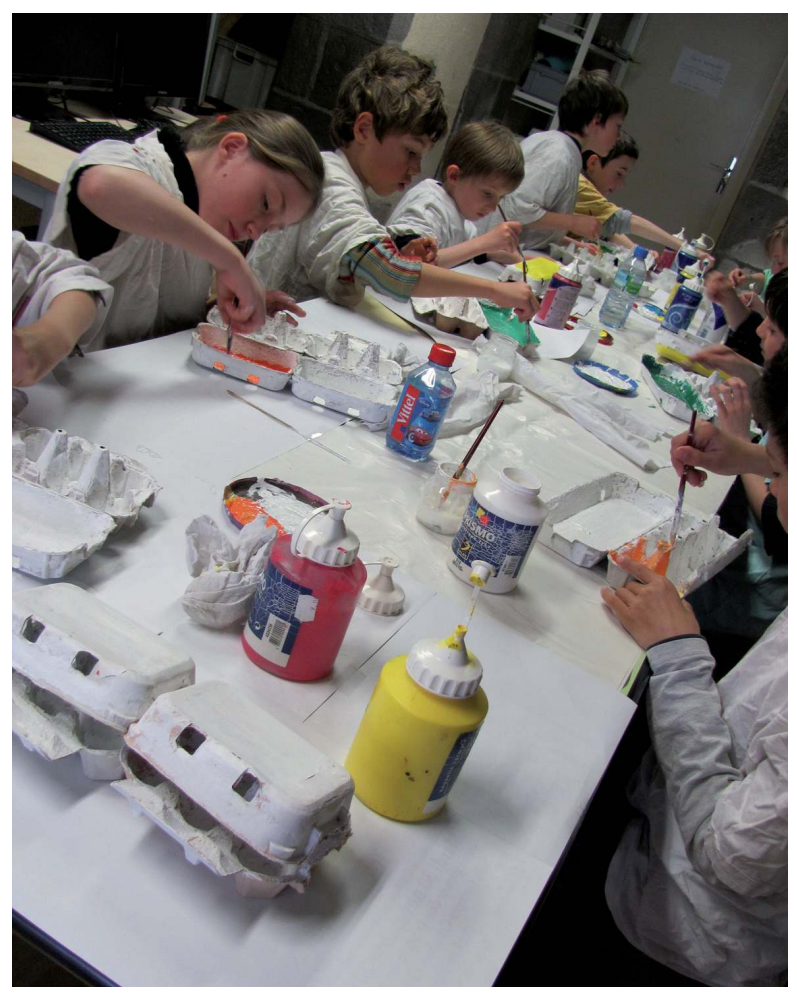

Atelier «Compte et compte et colégram » (Graine de science, avril 2012) : I'Awale est un jeu où il faut compter des graines et qui permet aux 8-12 ans de découvrir les machines et les objets à calculer, les chiffres et les nombres... et le respect de l'autre. ๑ Ville de Clermont-Ferrand/Muséum Henri-Lecoq/Aurélie Del Prete
Pour le service éducatif, habitué à travailler dans le domaine des sciences naturelles, la création de l'espace Blaise Pascal et l'élaboration d'outils pédagogiques ont nécessité de renouveler outils et méthodes de travail. Objets du patrimoine, reproduction fonctionnelle, reconstitution 3D, machine virtuelle et animations ont ainsi trouvé leur place dans la scénographie, mais aussi dans les outils pédagogiques à disposition de tous. Ces nouveaux outils, réfléchis par une équipe pluridisciplinaire, ont pour objectif d'allier explications et apports scientifiques, jeu et pédagogie.

C'est d'ailleurs avec la même idée qu'a été pensée et réalisée la soirée dédiée aux mathématiques « Voyage sur la math-monde » en mars 2013 au muséum Henri-Lecoq, dans le cadre de la semaine nationale des mathématiques. En effet, au cours de cette soirée, organisée et coanimée avec l'IREM, nous avons développé une approche ludique et interactive des mathématiques à partir de la découverte et de la manipulation d'instruments de calcul, de la Préhistoire à nos jours, et au travers de jeux mathématiques.

\section{Bibliographie}

Commission Inter IREM Instruments scientifiques à travers l'histoire. Éditions Ellipses, 2004.

Ellenberg, M. La machine à calculer de Blaise Pascal. Éditions Nathan. 1993.

Marguin, J. Histoire des instruments et machines à calculer, trois siècles de mécanique pensante, 1642-1942. Éditions Hermann, 1994.

Mourlevat, G. Les machines arithmétiques de Blaise Pascal. La française d'édition et d'Imprimerie, 1988

Vidal, N. et Vogt, D. Les machines arithmétiques de Blaise Pascal. Les collections du muséum Henri-Lecoq, vol. V, Histoire des sciences et techniques, 2011 .

\section{Sites Internet}

http://eduscol.education.fr/cid59178/semaine-des-mathematiques.html présentation de la semaine des mathématiques et bilan des éditions précédentes.

http://eduscol.education.fr/cid54958/mathc2.html : présentation du stage MathC2+ et bilan des éditions précédentes

http://musee.lecoq.clermont-ferrand.fr : site de la Ville de Clermont-Ferrand, pages du muséum Henri-Lecoq avec des fichiers pdf téléchargeables présentant les différents parcours-découverte et animations 